\title{
Gallium Oxide Nanoribbons and Nanosheets
}

\author{
Z. R. Dai, Z. W. Pan, and Z. L. Wang* \\ Center for Nanoscience and Nanotechnology, School of Materials Science and Engineering, \\ Georgia Institute of Technology, Atlanta, Georgia 30332-0245
}

Received: August 20, 2001; In Final Form: October 26, 2001

\begin{abstract}
Nanoribbons and flat nanosheets of $\mathrm{Ga}_{2} \mathrm{O}_{3}$ have been synthesized by evaporating $\mathrm{GaN}$ at high temperature with the presence of oxygen. The as-synthesized nanoribbons and nanosheets are pure, structurally uniform, single crystalline, and free from dislocations. The nanoribbons and the nanosheets all have monoclinic $\beta$ - $\mathrm{Ga}_{2} \mathrm{O}_{3}$ structure. The flat top and bottom surfaces for both nanoribbons and nanosheets are $\pm(100)$, the side surfaces are $\pm(010)$ and $\pm(10 \overline{1})$ for nanoribbons and $\pm(010), \pm(10 \overline{1})$ and $\pm(21 \overline{2})$ for nanosheets. The axis direction of nanoribbon growth is along either [001] or [010].
\end{abstract}

Investigation of the dimensionality and space confined transport phenomena in one-dimensional nanostructures has fundamental importance for nanoelectronics and optoelectronics. Carbon nanotubes are a model system for systematic studies on the electrical, optical, and thermal transport phenomena in one-dimensional objects. In addition to carbon nanotubes, wirelike nanostructures of various materials such as carbides $\left(\mathrm{SiC},{ }^{1-3}\right.$ $\mathrm{TiC}^{1}$ ), nitrides $\left(\mathrm{GaN},{ }^{4-7} \mathrm{Si}_{3} \mathrm{~N}_{4}{ }^{8}\right)$, semiconductor compounds $\left(\mathrm{GaAs},{ }^{9} \mathrm{InAs}^{10}\right)$, element semiconductors $\left(\mathrm{Si},{ }^{11-14} \mathrm{Ge}^{15,16}\right)$, and dielectric $\mathrm{Ga}_{2} \mathrm{O}_{3}$ nanowires ${ }^{17}$ have been synthesized. These nanowires have a common cylindrical symmetric cross-section that is sometime sheathed by a thin amorphous oxide layer. Recently, we have synthesized belt-like nanostructures of semiconducting oxides of zinc, tin, indium, cadmium, and lead. ${ }^{18-20}$ The as-synthesized oxide nanobelts are pure with high crystallinity and most are free from dislocations. They have a rectangular-like cross-section with typical widths of 30-300 $\mathrm{nm}$, width-to-thickness ratios of 5-10, and lengths of up to a few millimeters.

Gallium oxide is an important functional material. It has been used as insulating barrier for spin-dependent tunneling junction. ${ }^{21}$ Manganese-activated gallium oxide has been demonstrated as an electroluminescent phosphor. ${ }^{22}$ Crystalline gallium oxide nanowires has been found to give blue light emission. ${ }^{23}$ In this paper, we report the synthesis and characterization of nanoribbons and nanosheets of $\mathrm{Ga}_{2} \mathrm{O}_{3}$, of which morphology and structure appear to be distinctively different from the ones reported in the literature.

Our synthesis is based on thermal evaporation of $\mathrm{GaN}$ powders under controlled conditions with the presence of oxygen. The commercially supplied $\mathrm{GaN}$ powders $(99.99 \%$ metals basis, Alfa Aesar) were placed at the center of an alumina tube that was inserted in a horizontal tube furnace. The furnace was heated at a rate of $10-15^{\circ} \mathrm{C} / \mathrm{min}$ to $1100{ }^{\circ} \mathrm{C}$, and then maintained at this temperature for $2 \mathrm{~h}$ before being cooled to room temperature. The pressure in the growth chamber was 300 Torr, and the Ar flowing rate was $50 \mathrm{sccm}$. During evaporation, the products were deposited onto polycrystalline alumina plates placed at the downstream end of the alumina tube, where the temperature is about $800-850{ }^{\circ} \mathrm{C}$.

* Corresponding author. E-mail: zhong.wang@mse.gatech.edu.
The as-deposited products are wool-like with a light gray color. The structure was analyzed by X-ray diffraction (XRD) (Philips PW 1800 with $\mathrm{Cu} \mathrm{K} \alpha$ radiation), scanning electron microscopy (SEM) (Hitachi S800 FEG), transmission electron microscopy (TEM) (Hitachi HF-2000 FEG at $200 \mathrm{kV}$ and JEOL 4000EX high-resolution TEM (HRTEM) at $400 \mathrm{kV}$ ), and energy-dispersive X-ray spectroscopy (EDS).

SEM observations reveal that the products consist of a large quantity of wire-like nanostructures with typical lengths in the range of several tens to several hundreds of micrometers; some of them even have lengths on the order of millimeters (Figure 1). The as-synthesized sample also contains a large fraction of sheet-type structures, and size of the sheets is about $10 \mu \mathrm{m}$ across and several tens of nanometers in thickness. The nanosheets have straight edges with sharp corners, suggesting that they terminate by faceted crystallographic planes. Chemical microanalysis using EDS has showed that the nanostructures obtained are $\mathrm{Ga}_{2} \mathrm{O}_{3}$. The formation of $\mathrm{Ga}_{2} \mathrm{O}_{3}$ rather than $\mathrm{GaN}$ is believed due to the high combinability of $\mathrm{Ga}$ with oxygen, which is believed to leak into the growth tube.

Figure 2A gives a low magnification TEM image showing the ribbonlike structure of $\mathrm{Ga}_{2} \mathrm{O}_{3}$. The waving and twist shapes of the ribbons are apparent. The width of the ribbons varies from 40 to $300 \mathrm{~nm}$, and the geometry of the ribbons is rather uniform. The thickness of the ribbons is 10 to $60 \mathrm{~nm}$ depending on the width. The contrast observed over the ribbon is bending contour due to a bending of the ribbon with respect to electron beams (Figure 2B). This is an electron diffraction phenomenon and is most frequently observed in metal foils due to deformation and bending. ${ }^{24}$ The nanoribbons are single crystalline and free from dislocations. Their surfaces are clean without an amorphous layer.

$\mathrm{Ga}_{2} \mathrm{O}_{3}$ is of polymorphism in its crystal structure. The polymorph of $\mathrm{Ga}_{2} \mathrm{O}_{3}$ mainly includes $\alpha-(R \overline{3} m, a=4.979 \AA$ and $\left.c=13.429 \AA^{25}\right), \beta$ - $(C 2 / m, a=12.23, b=3.04, c=5.80$ $\AA$, and $\left.\beta=103.7^{\circ}{ }^{26}\right)$, and $\gamma-\mathrm{Ga}_{2} \mathrm{O}_{3}\left(F d \overline{3} m, a=8.22 \AA^{27}\right)$. Of those phases, the $\beta-\mathrm{Ga}_{2} \mathrm{O}_{3}$ is only stable modification. ${ }^{28}$ Figure $2 \mathrm{C}$ is an electron diffraction pattern recorded from the nanoribbon displayed in Figure 2B. Carefully measuring spacing distances of the hexagon-like reflection patterns indicates that the spacing corresponding to the reflection " $a$ " is slightly shorter than that for both reflections " $b$ " and " $c$ " while those of the 

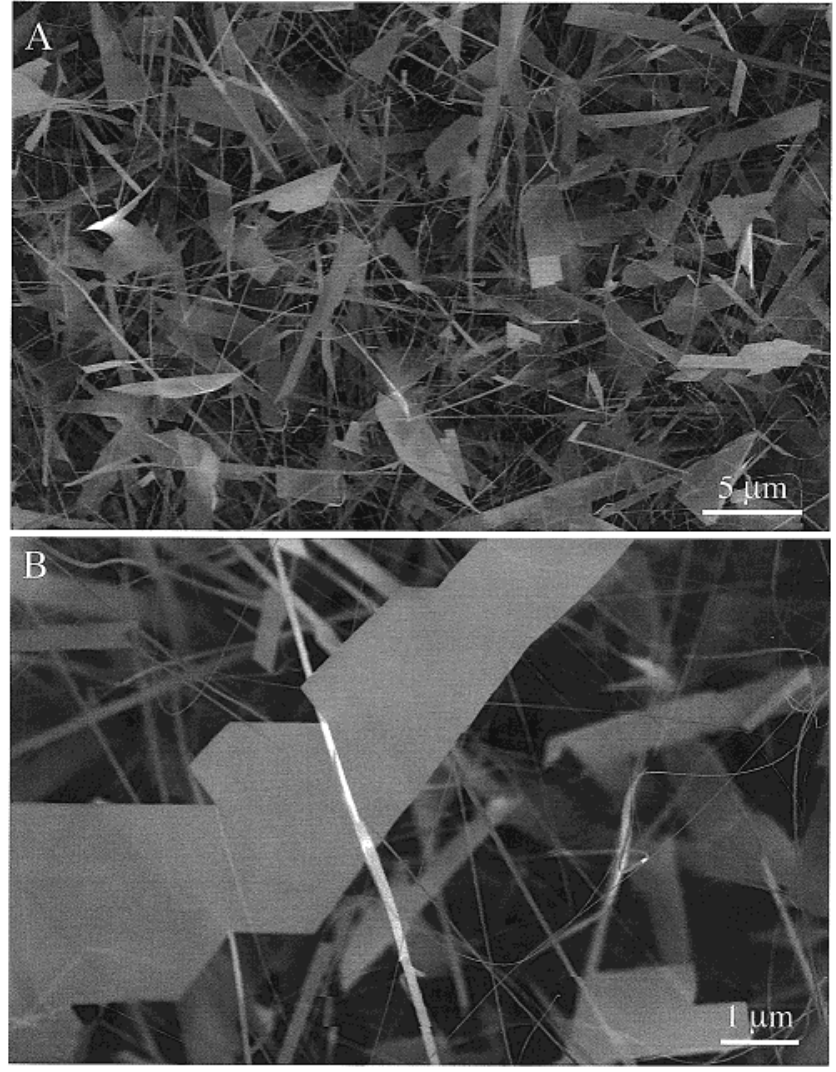

Figure 1. SEM images of $\mathrm{Ga}_{2} \mathrm{O}_{3}$ nanoribbon and nanosheets recorded at $15 \mathrm{kV}$ without surface coating.

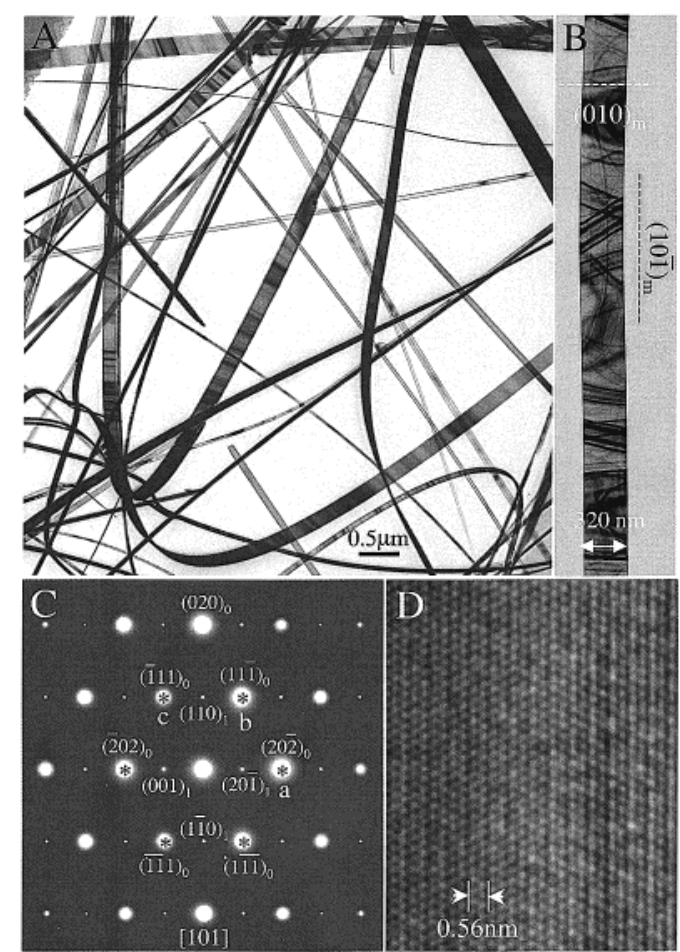

Figure 2. (A) Low-magnification TEM image of $\mathrm{Ga}_{2} \mathrm{O}_{3}$ nanoribbons. (B) A nanoribbon lying on the carbon substrate; (C) the corresponding electron diffraction pattern, which is indexed to be the [101] of $\beta-\mathrm{Ga}_{2} \mathrm{O}_{3}$; and (D) a HRTEM image.

latter two are equal. The configuration composing the asterisks virtually is not a regular hexagon, concluding that the crystal structure cannot be a cubic lattice. By checking the included angles of the reflections and their corresponding spacing distances of crystal planes, the reflections with strong intensity are determined to belong to the [101] crystal zone of $\beta-\mathrm{Ga}_{2} \mathrm{O}_{3}$ phase. The corresponding crystal plane indexes are marked in the electron diffraction pattern (Figure 2C), where the subscript " 0 " represents zero-order Laue zone reflection. Some extra weaker reflections, however, are also clearly seen, which are located at the reflection extinctive positions of [101] crystal zone of $\beta-\mathrm{Ga}_{2} \mathrm{O}_{3}$ due to the symmetry corresponding to the space group $C 2 / m$ (its reflection condition is $h+k=2 n$ ). Those weaker reflections are identified to be high-order (1st-order) Laue reflection. The corresponding crystal indexes are labeled with a subscript " 1 ". Appearance of the high-order Laue zone reflections is due to two reasons. One is that the thickness of the $\beta-\mathrm{Ga}_{2} \mathrm{O}_{3}$ nanoribbon is very thin, resulting in long reciprocal rods shape factor along the beam direction, which can be cut by the Ewald sphere. Second, for the beam direction of [101], the $(101)^{*}$ reciprocal plane (in reciprocal space) is closely parallel to the (100) crystal plane, but the real space spacing of (100) planes is rather large, thus, the distance between the (100) reflections is rather small in reciprocal. Considering the elongated shape factor of the reciprocal lattice rod along the beam direction due to the small thickness of the nanoribbon, the Ewald sphere could cut through the first-order Laue zone, resulting in the weak reflection spots in the diffraction pattern, along with the strong zero-order Laue reflections. This result was confirmed experimentally using the samples with different thickness. The nanoribbons are enclosed by $\pm(100)$ top and bottom surfaces, and $\pm(010)$ and $\pm(10 \overline{1})$ side surfaces. It has been found that both the $\pm(010)$ and the $\pm(10 \overline{1})$ crystal planes can serve as growth front of nanoribbons with growth directions of [010] and [001], respectively. Figure 2B shows an example where the growth front of the nanoribbon is $\pm(010)$. A corresponding HRTEM image is shown in Figure 2D, which indicates a perfect crystallinity of the $\mathrm{Ga}_{2} \mathrm{O}_{3}$ nanoribbon.

Nanosheets have some straight edges with corners of specific angles, typically of $45^{\circ}$ and $90^{\circ}$ (Figure 3). Some of the nanosheets are directly connected to nanoribbons (Figure 3B), indicating that they have the same structure. Electron diffraction shows that the sheets have the same crystal structure of $\beta-\mathrm{Ga}_{2} \mathrm{O}_{3}$ as that of nanoribbons. The inserted electron diffraction pattern (Figure 3C) is the same as that shown in Figure 2C, which correspond to the [101] crystal zone of $\beta-\mathrm{Ga}_{2} \mathrm{O}_{3}$. The intensity distribution of reflections in the pattern inserted in Figure $3 \mathrm{C}$, however, is rather different from that in Figure $2 \mathrm{C}$, which is due to the fact that electron beam is not exactly parallel to the [101] zone axis for the pattern in Figure 3C. This further verifies that the extra reflections for [101] crystal zone are associated with high-order Laue reflection effect. The two perpendicular planes result in the rectangular- and L-shape structures (Figure $3 \mathrm{~A})$. Beside the $\pm(010)$ and the $\pm(10 \overline{1})$ facets, a third-type of planes with a $\sim 45^{\circ}$ angle with the two sides, as indicated by arrowheads in Figure 3A, is also observed. This type of planes is identified to be the $(21 \overline{2})$ plane from the electron diffraction pattern. The top and bottom surfaces are also the $\pm(100)$ crystal planes of $\beta-\mathrm{Ga}_{2} \mathrm{O}_{3}$. The contrast of the sheet presented in Figure $3 \mathrm{C}$ is complex due to the bending of the ceramic foil. The flexibility of the ceramics increases dramatically as its size reduces. This is different from bulk ceramics. Based on our SEM observation, the thickness of the nanoribbons and nanosheets are $20-60 \mathrm{~nm}$.

The growth morphology of nanoribbons and nanosheets reported here are distinctively different from that of $\mathrm{Ga}_{2} \mathrm{O}_{3}$ nanorods ${ }^{29}$ and nanowires. ${ }^{30}$ The nanorods grown by thermal evaporation of a mixture of $\mathrm{GaN}$, graphite, and Ni particles have 


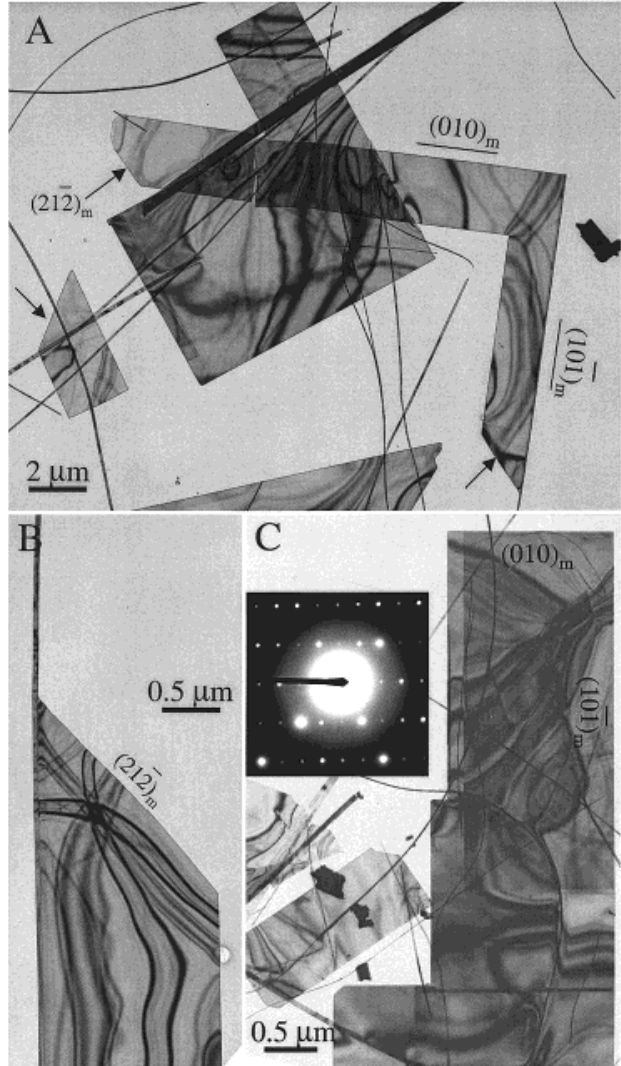

Figure 3. Low-magnification TEM images of $\beta-\mathrm{Ga}_{2} \mathrm{O}_{3}$ nanosheets. The inset in $(\mathrm{C})$ is the corresponding electron diffraction pattern recorded from the large nanosheet.

diameters of 5-10 $\mathrm{nm}$ and growth directions of either [111] or [200]. The nanowires grown by an arc-discharge of GaN with the presence of $\mathrm{Co}$ and $\mathrm{Ni}$ particles have diameters of 20-30 $\mathrm{nm}$ and growth direction of [001].

The unique structure of the nanoribbons and nanosheets apparently indicates that their growth may be largely determined by growth kinetics. There are two common growth mechanisms for nanowires. The vapor-liquid-solid (VLS) process ${ }^{31}$ was proposed for the nanowires grown by a catalytic assisted technique, ${ }^{11}$ in which a metal particle is located at the growth front of the wire and acts as the catalytic active site. In the vapor-solid (VS) process, ${ }^{32}$ the oxide vapor, evaporated from the starting oxide at a higher temperature zone, directly deposits on a substrate at a lower temperature region and grows into ribbonlike nanostructures. The growth of the nanoribbons and nanosheets might be dominated by the VS process, but we cannot totally rule out the VLS process because low melting point Ga metal particles are reduced in the synthesis, which may act as catalyst for the growth. The formation of nanoribbons and nanosheets is a combined result of VLS and VS as well as growth kinetics.
Acknowledgment. The authors are thankful for the support from NSF Grants DMR-9733160 and the Georgia Tech Electron Microscopy Center.

\section{References and Notes}

(1) Dai, H. J.; Wong, E. W.; Lu, Y. Z.; Fan, S. S.; Lieber, C. M. Nature 1995, 375, 769.

(2) Pan, Z. W.; Lai, H. L.; Au, F. C. K.; Duan, X. F.; Zhou, W. Y.; Shi, W. S.; Wang, N.; Lee, C. S.; Wong, N. B.; Lee, S. T.; Xie, S. S. Adv Mater. 2000, 12, 1186.

(3) Wang, Z. L.; Dai, Z. R.; Gao, R. P.; Bai, Z. G.; Gole, J. L. Appl. Phys. Lett. 2000, 77, 3349. 1287.

(4) Han, W. Q.; Fan, S. S.; Li, Q. Q.; Hu, Y. D. Science 1997, 277,

(5) Duan, X. F.; Lieber, C. M. J. Am. Chem. Soc. 2000, 122, 188.

(6) Chen, C. C.; Yeh, C. C. Adv. Mater. 2000, 12, 738.

(7) Chen, C. C.; Yeh, C. C.; Chen, C. H.; Yu, M. Y.; Liu, H. L.; Wu, J. J.; Chen, K. H.; Chen, L. C.; Peng, J. Y.; Chen, Y. F. J. Am. Chem. Soc. 2001, 123, 2791.

(8) Han, W. Q.; Fan, S. S.; Li, Q. Q.; Gu, B. L.; Zhang, X. B.; Yu, D. P. Appl. Phys. Lett. 1997, 71, 2271. 1116.

(9) Duan, X. F.; Wang, J. F.; Lieber, C. M. Appl. Phys. Lett. 2000, 76,

(10) Li, H. X.; Wu, J.; Wang, Z. G.; Daniels-Race, T. Appl. Phys. Lett. 1999, 75,1173 .

(11) Morales, A. M.; Lieber, C. M. Science 1998, 279, 208.

(12) Yu, D. P.; Bai, Z. G.; Feng, S. Q.; Lee, C. S.; Bello, I.; Sun, X. S.; Tang, Y. H.; Zhou, G. W.; Zhang, Z. Solid State Commun. 1998, 105, 403.

(13) Lee, S. T.; Wang, N.; Zhang, Y. F.; Tang, Y. H. MRS Bull. 1999, $24,36$.

(14) Pan, Z. W.; Dai, Z. R.; Xu, L.; Lee, S. T.; Wang, Z. L. J. Phys. Chem. 2001, B105, 2507.

(15) Wu, Y.; Yang, P. Chem. Mater. 2000, 12, 605.

(16) Zhang, Y. F.; Tang, Y. H.; Wang, N.; Lee, C. S.; Bello, I.; Lee, S. T. Phys. Rev. 2000, B 61, 4518.

(17) Choi, Y. C.; Kim, W. S.; Park, Y. S.; Lee, S. M.; Bae, D. J.; Lee, Y. H.; Park, G. S.; Choi, W. B.; Lee, N. S.; Kim, J. M. Adv. Mater. 2000, $12,746$.

(18) Pan, Z. W.; Dai, Z. R.; Wang, Z. L. Science 2001, 291, 1947.

(19) Dai, Z. R.; Pan, Z. W.; Wang, Z. L. Solid State Commun. 2001, 118,351 .

(20) Pan, Z. W.; Dai, Z. R.; Wang, Z. L. Appl. Phys. Lett. 2000, submitted.

(21) Li, Z.; de Groot, C.; Moodera, J. H. Appl. Phys. Lett. 2000, 77, 3630 .

(22) Miyata, T.; Nakatani, T.; Minami, T. Thin Solid Films 2000, 373, 145 .

(23) Wu, W. C.; Song, W. H.; Huang, W. D.; Pu, M. H.; Zhao, B.; Sun, Y. P.; Du, J. J. Chem. Phys. Letts. 2000, 328, 5.

(24) Hirsch, P. B.; Howie, A.; Nicholson, R. B.; Pashley, D. W.; Whelan, M. J. Electron Microscopy of Thin Crystals; Butterworth: 1965.

(25) Swanson, H. E.; Fuyat, R. K.; Ugrinie, G. M. Natl. Bur. Stand. Cric. 1955, 539 IV, 25

(26) Geller, S. J. Chem. Phys. 1960, 33, 676.

(27) Pohl, K. Naturwissenschaften 1968, 55, 82.

(28) Schneider, S. J.; Waring, J. L. J. Res. Nat. Bur. Stand. 1963, A67, 19

(29) Park, G. S.; Choi, W. B.; Kim, J. M.; Choi, Y. C.; Lee, Y. H.; Lim, C. B. J. Cryst. Growth 2000, 220, 494.

(30) Han, W. Q.; Kohler-Redlich, P.; Ernst, F.; Ruhle, M. Solid State Commun. 2000, 115, 527.

(31) Wagner, R. S.; Ellis, W. C. Appl. Phys. Lett. 1964, 4, 89.

(32) Brenner, S. S.; Sears, G. W. Acta Met. 1956, 4, 268. 